\title{
Kyste odontogène calcifié : à propos d'un cas clinique
}

\section{Cyst odontogene calcified: about a clinical case}

\section{MOTS-CLEFS :}

- Kyste, cavité orale, tumeur bénigne

KEYWORDS:

- Cyst, oral cavity, benign tumor

$\operatorname{AOS} n^{\circ} 287-2018$

\section{Résumé}

Le kyste odontogéne calcifié est une lésion bénigne rare, décrite pour la première fois par Gorlin en 1962, et classée comme tel dans la classification de 1992.

Il resprésente $2 \%$ des tumeurs odontogènes, il peut apparaitre ò n'importe quel age, avec une prédominance du sexe masculin

Les aspects cliniques et radiologiques de cette lésion ne sont pas spécifiques, la confirmation $\mathrm{du}$ diagnostic et anatomopathologique.

$\grave{A}$ travers un cas cliniques d'un kyste odontogène calcifié, ce travail permet d'illustrer les spécificités de cette lésion
Abstract

The calcified odontogenic cyst is a rare benign lesion, described for the first time by Gorlin in 1962 and classified as such in the classification of 1992.

It present $2 \%$ of odontogenic tumors, it can appear at any age, predominantly male

Clinical and radiological aspects of this lesion is not specific, confirmation of the diagnosis is histological.

Through a clinical case of a calcified odontogenic cyst, this work illustrates the characteristics of this lesion

\section{Mohammed FAROUK, Service d'odontologie chirurgicale, Centre de consultation
et traitement dentaire, CHU Ibn Rochd, Casablanca, Maroc.
Olaya MEDAGHRI ALAOUI, Service d'odontologie chirurgicale, Centre
de consultation et traitement dentaire, CHU Ibn Rochd, Casablanca, Maroc.
Ihsane BEN YAHYA, Service d'odontologie chirurgicale, Centre de consultation
et traitement dentaire, CHU Ibn Rochd, Casablanca, Maroc.}

\section{INTRODUCTION}

Le kyste odontogène calcifié est une lésion bénigne rare, décrite pour la première fois par Gorlin en 1962, et classée comme tel dans la classification de 1992. Cependant, en 2005 l'OMS a classé cette lésion en tant que tumeur odontogène calcifié.

\section{OBSERVATION}

Un patient âgé de 50 ans s'est présenté à la consultation pour une tuméfaction palatine droite évoluant depuis 10 ans. Le patient rapportait que l'évolution était asymptomatique, et qu'il a eu plusieurs traitements médicaux sans aucune amélioration.
L'examen exobuccal était sans spécificité.

L'examen endobuccal a montré la présence au niveau de la moitié droite du palais, d'une tuméfaction ferme à la palpation et indolore. la muqueuse de recouvrement était d'aspect normal. Aucun signe n'a été detecté en vestibulaire (Fig. 1a, b).

Le test de vitalité était négatif pour les 13, 15.

L'examen radiologique rétro alvéolaire a montré la présence, au niveau du secteur prémolaire maxillaire droit, d'une lésion mixte sans rapport avec les 13, 15, la lésion n'était pas totalement visible sur la rétro-alvéolaire ce qui a nécessité le recours à la panoramique (Fig. 2a, b, c). Sur la panoramique la lésion était radioclaire et centrée par une image radio-opaque, sans rapports avec les dents. L'examen cône beam a montré une soufflure des corticales internes et externes, sans envahissement sinusal. 


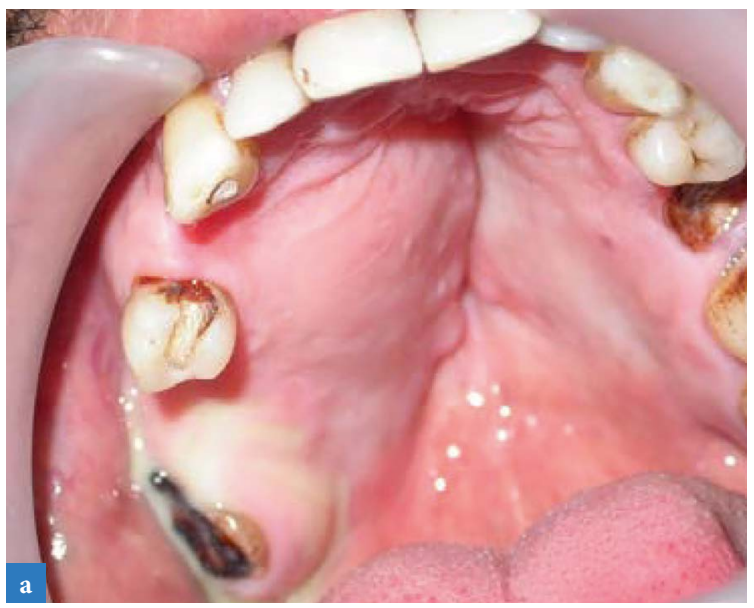

$\triangle$ Fig. 1a : Tuméfaction palatine.

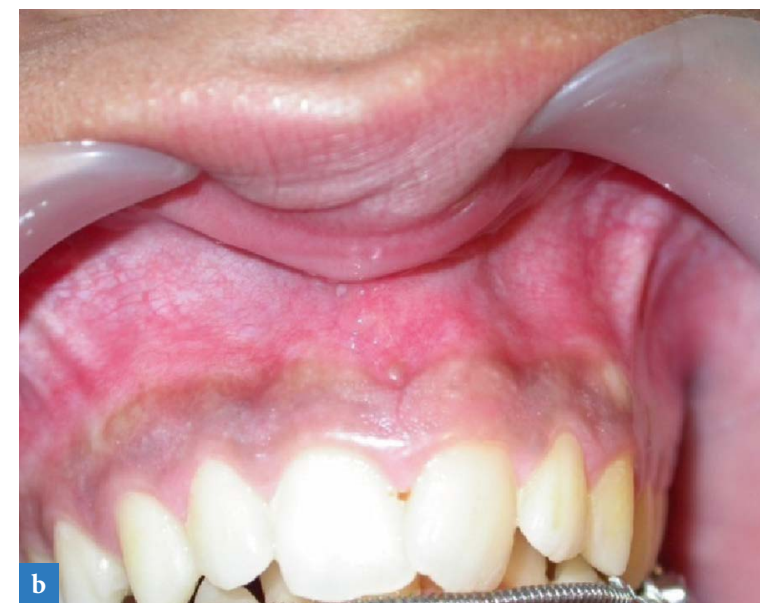

$\triangle$ Fig. 1b : Absence de signes en vestibulaire.

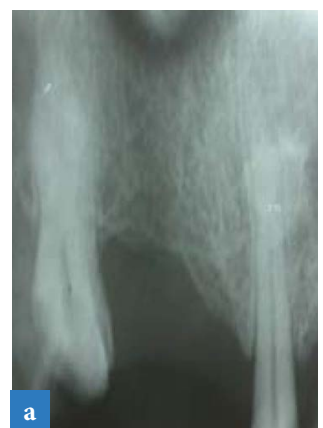

$\triangle$ Fig. 2a : Retroalvéolaire.

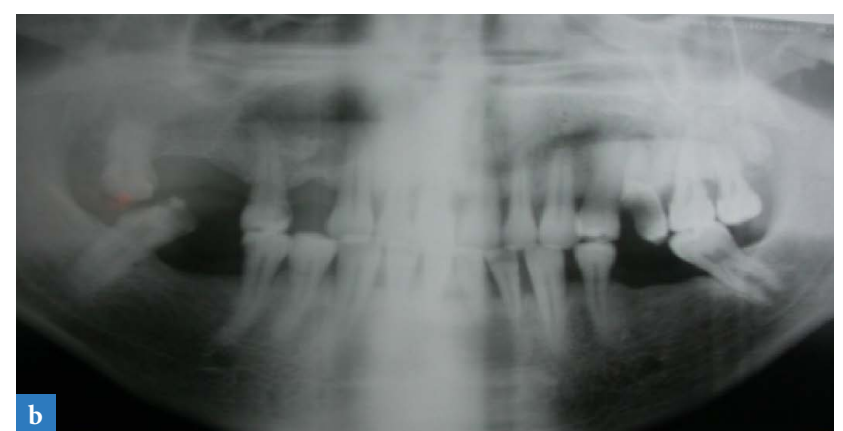

$\triangle$ Fig. 2 b : La panoramique.

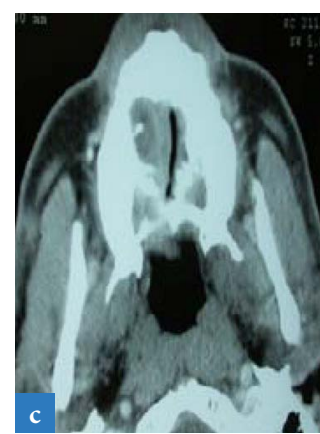

$\triangle$ Fig. 2c : Cône beam.
Suite à ces examens deux diagnostics ont été evoqués : le kyste inflamatoire, kyste odontogène calcifié.

Le traitement endodontique a été rélisé pour les 13, 15. La prise en charge a consisté en une énucléation du kyste, l'abord de la lésion était palatin vu sa localisation. Deux particles solides noirâtres ont été contenues dans la lésion (Fig. 3a, 3b).

L'examen anatomopathologique a montré que la paroi kystique est formée d'une épithélium riche en cellules inflammatoire avec présence de cellules améloblastiques au niveau de la couche interne, les deux corps solides sont formés par des tissus dentaires mixtes (Fig. 4).

Suite à l'examen anatomopathologique, le diagnostic du kyste odotogène calcifié a été retenu.

Le contrôle effectué après deux mois montre une cicatrisation du site, associée à une régénération osseuse au niveau de la cavité kystique (Fig. 5a, 5b).

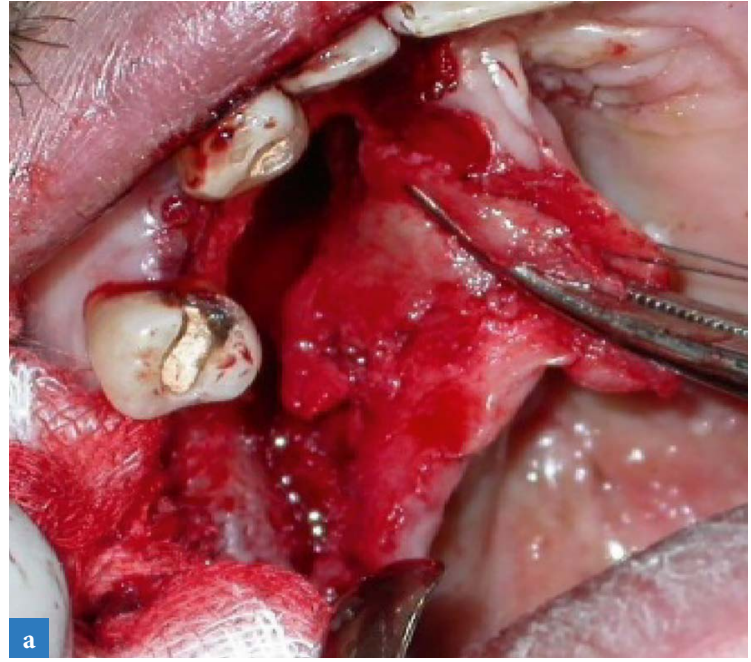

$\triangle$ Fig. 3a : L'énucléation.

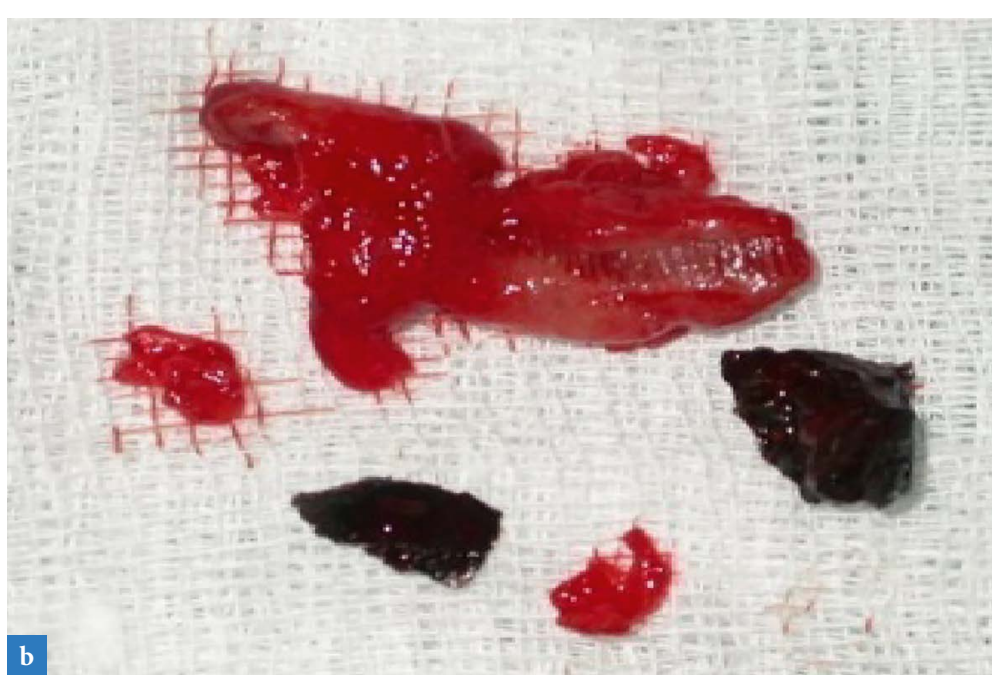

$\triangle$ Fig. 3b : La pièce opératoire. 

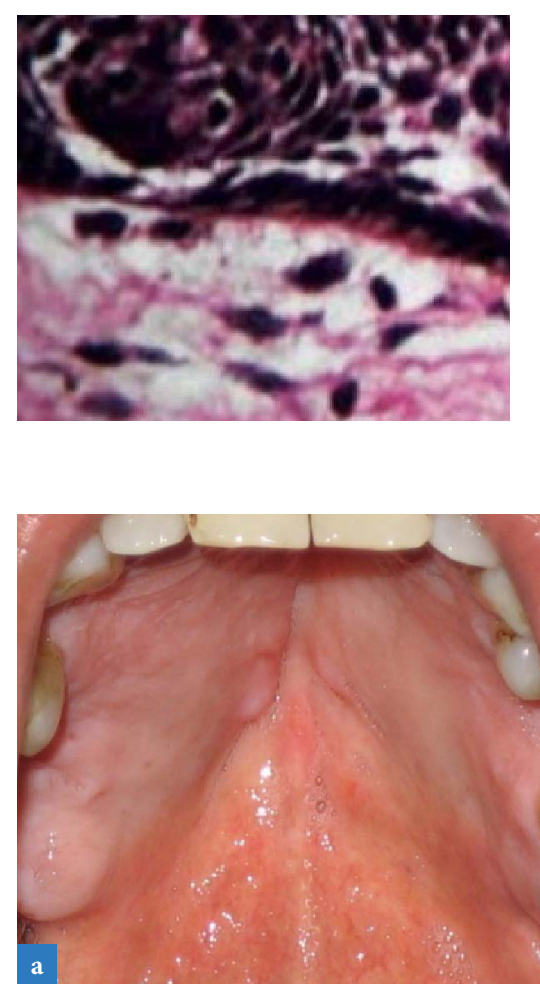

$\triangle$ Fig. 5a : Le contrôle clinique.
$<$ Fig. 4: L'examen anatomopathologique montre une paroi kystique riche en cellules améloblastique au niveau de la couche interne.

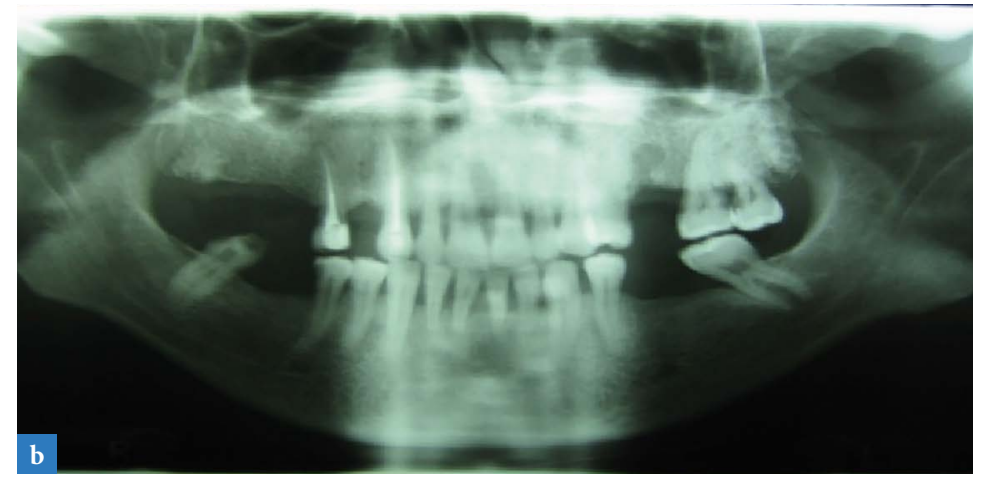

$\triangle$ Fig. 5b : Le contrôle radiologique.

\section{DISCUSSION}

En 2005 L'OMS a classée le kyste odontogène calcifié en tant que tumeur, ainsi la nouvelle nomonclature de cette entité est la tumeur kystique odontogène calcifiée $[1,2]$.

Il resprésente $2 \%$ des tumeurs odontogènes, il peut apparaitre à n'importe quel âge [3], avec une prédominance du sexe masculin [4].

Le siége peut être intra ou extra-osseux. Les deux formes centrales et périphériques se développent avec une fréquence égale au maxillaire et à la mandibule avec une majorité des lésions situées dans les secteurs incisivo-canins [2,3], ce qui correspond au cas rapporté ou la lésion était située au niveau canin maxillaire.

Le KOC central apparaît comme une tuméfaction asymptomatique associée à une lyse osseuse adjacente, alors que, dans sa variante périphérique, il se présente comme une formation sessile bien circonscrite non spécifique, ou une masse pédiculée, avec une surface lisse $[2,3]$.

Le KOC est généralement asymptomatique [5, 6], mais si la localisation est maxillaire, les patients peuvent parfois se plaindre d'obstructions nasales, d'épistaxis et de maux de tête [7].

Le KOC est une tumeur à évolution lente et progressive, et donc l'aspect radiologique est différent en fonction du stade d'évolution $[7,8]$.
Au début la lésion peut se présenter sous forme d'une image radioclaire uni ou multiloculaire bien limitée. À un stade avancé la lésion apparaît comme une image mixte [8].

Radiologiquement cet aspect mixte n'est pas standard mais il existe dans la moitié des cas [9].

Des radio-opacités apparaissent à l'intérieur du kyste dues à la formation de particules calcifiées plus ou moins régulières, qui peuvent avoir la forme de dents [3], de taille et de nombre variables [8]. Les racines des dents adjacentes sont décalées ou résorbés par l'expansion de la lésion. L'os cortical est aminci, expansé ou perforé $[3,8,9]$.

La tomodensitométrie permet de visualiser la matrice tumorale, et de déterminer l'extension de la tumeur, ses rapports avec les structures anatomiques voisines. Au niveau osseux péri-tumoral, on note des images de condensation $[8,9]$.

L'aspect histologique est spécifique, l'épihélium kystique est caractérisé par la présence d'une couche externe de cellules cylindriques et d'une couche interne formée par des cellules améloblastiques et une capsule fibreuse. La présence de calcifications et de cellules dites fantomes éparpillées dans l'épithélium est spécifique à cette lésion. Ces cellules peuvent se calcifier d'où l'origine de formation des calcifications.

Le mécanisme de formation de cellules fantômes est controversé, il peut être du à une nécrose de coagulation ou une forme de kératinisation normale 
ou aberrante de l'épithélium odontogène. Certaines théories révèlent un processus ischémique sous-jacent, ce qui peut entraîner une métaplasie squameuse et plus tard une calcification $[2,9]$.

Le diagnostic différentiel dépend du stade de l'évolution. Au stade de radioclarté, le KOC peut ressembler à un améloblastome, un fibrome améloblastique, un kyste épidermoïde, un kyste folliculaire, voire un kyste péri-apical. Après l'apparition de ces calcifications, le diagnostic différentiel peut se poser avec un cémentome, un odontome, ou une dysplasie $[3,9]$.

Le KOC est souvent associé à des dents incluses ou des odontomes [7].

L'énucléation est le traitement de choix pour la plupart des KOC intra-osseux, l'exérèse pour la forme extra-osseuse [10].

Le taux de récidive est très bas $5 \%$. La transformation maligne est très rare [8].

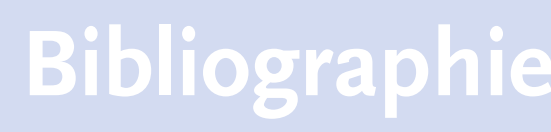

[1] Hamdi H, Ben Alaya T, Ben Romdhame I, Khatteche B, Favre de Thierrens C. La tumeur épithéliale odontogénique calcifiée : à propos d'un cas. Med Buccale Chir Buccale 2002; 8(2): 87-90.

[2] Zornosa X, Müller S. Calcifying Cystic Odontogenic Tumor, Head Neck Pathol 2010; 4(4): 292-294.

[3] Kler S, Palaskar S, Shetty VP, Bhushan A. Intraosseous calcifying cystic odontogenic tumor. J Oral Maxillofac Pathol 2009; 13(1): 27-29.
[4] Pereira CM,Carneiro DS, Pofahl HBC, Gasparetto PF, Silva Junior AF. Calcifying cystic odontogenic tumor radiographically mimicking a lateral periodontal cyst: clinical case report. J Health Sci Inst 2010; 28(4): 315-7.

[5] Utumi ER, Pedron IG, Nucci da Silva LP, Machado GG, Rocha AC. Different manifestations of calcifying cystic odontogenic tumor. Einstein 2012; 10(3): 366-70.

[6] Marques YMFS, Botelho TL, Xavier FCA, Rangel AL,Rege ICC, Mantesso A. Importance of cone beam computed tomography for diagnosis of calcifying cystic odontogenic tumor associated to odontoma. Report of a case. Med Oral Patol Oral Cir Bucal 2010; 15(3): 490-3.
[7] Kelly R. Magliocca, M.D. Odontogenic cysts calcifying odontogenic. Cyst Oral Surg Oral Med Oral Pathol 1975; 39: 769-772.

[8] George R, Donald PM, Sabarinath B. Calcifying cystic odontogenic tumor: A case report and review. IJSS Case Reports \& Reviews 2015; 15(8): 128-132.

[9] Gamoh S, Nakashima Y, Akiyama H. A unique case of a calcifying cystic odontogenic tumor. Open J Stomatol 2013; 3: 314-317.

[10] Prashant K, Pandilwar U. Calcifying epithelial odontogenic cyst associated with complex odontome of maxilla. J Maxillofac Oral Surg 2013; 12(1): 85-89. 